\title{
The Impact of Commercial Open Source Software on Proprietary Software Producers and Social Welfare
}

\author{
Mingqing Xing \\ School of Economics and Management and Neural Decision Science Laboratory, Weifang University (China)
}

mqxing1979@163.com

Received: September 2014

Accepted: November 2014

\section{Abstract:}

Purpose: A growing number of commercial open source software, based on community open source, appears in many segments of the software market. The purpose of this study is to investigate how commercial open source software affects the pricing (market share or profit) of proprietary software producer, consumer surplus and social welfare.

Design/methodology: To analyze the impact of commercial open source software on proprietary software producer, this study constructs two vertical-differentiation models: the basic model considers proprietary software only competing with community open source software, and its extended one considers proprietary software competing with both community and commercial open source software.

Findings: This study mainly finds that the presence of commercial open source software can lead to the decrease of the software price and profit for proprietary software producer, while the consumer surplus and social welfare will be increased. However, it does not necessarily cause the decline in the market share for proprietary software producer.

Originality/value: The main contribution of this study is to examine the effect of commercial open source software on the competitive strategies of proprietary software producer, consumer surplus and social welfare. 
Keywords: competition, open source software, commercial open source software, community open source software, proprietary software, software usability

\section{Introduction}

The rapid development of open source software (e.g., Linux) is a significant phenomenon in the software industry. Open source software (OSS) allows software developers to share its software source codes, identify and correct errors, and redistribute its software source codes (O'Reilly's, 1999; Coar, 2006). Open source software has become a threat to proprietary software in many segments of the software market. In server operating system market, Linux, as an open source operating system, holds more than a $30 \%$ share, and Microsoft's Windows holds approximately a $50 \%$ share. In web server market, more than $60 \%$ of websites use Apache (an open source software), but only about 30\% employ Microsoft's Internet Information (Lin, 2008). This attracts the interest of scholars. Raghunathan, Prasad, Mishra and Chang (2005), Economides and Katsamakas (2006), Choudhary and Zhou (2007), Sen (2007), Jaisingh, See-To and Tam (2008), Lee and Mendelson (2008), Lanzi (2009), Xing (2010a), Chen, Liu and Tang (2011), Zhu and Zhou (2012), Zeroukhi and Pénard (2014) and Gramstad (2014) study the competition between open source and proprietary software. However, their research only considers community (or free) open source but does not take into account commercial open source.

Open source software can be made a fundamental distinction between community and commercial open source (Riehle, 2012). The former is owned by a not-for-profit community, whose members generally do not derive direct revenues from this software. In contrast, the latter is owned by a software vendor, whose purpose is to get revenues from this software. In recent years, more and more firms build commercial products on the basis of open source software. The total amount of work invested in open source projects is growing exponentially (Deshpande and Riehle, 2008). According to Kumar, Gordon and Srinivasan (2011), commercial open source software is privately developed software product based on the publicly available source codes. For example, Red Hat Inc's commercial versions of the free available Linux are designed to improve the usability of the Linux operating system. Many scholars describe the commercial use of open source software and provide the commercial open source business models. For examples, Dixon (2009) gives the beekeeper model of commercial open source software and thinks that this model is a perfect combination of the open source project and the software firm supporting that project, and a complete 'go to market' program; Riehle (2012) investigates the essential properties of commercial open source business models and shows how firms using these models can access to market faster with a better product at lower operating costs than possible for traditional competitors; Popp (2012) analyzes how the hybrid business model of commercial open source leverages licensing strategies and open source 
communities to create value for software venders and consumers; Widenius and Nyman (2014) study what motivates businesses to get to participate in open source and the issues regarding the monetization of open source projects. Some authors focus on the innovation incentives for commercial open source providers. For examples, Kumar et al. (2011) examine the competition between commercial open source firms and answer why a firm further develops a software product if the competitors can free ride on its contributions; Xing (2013) compares commercial open source companies' innovation incentives under the General Public License (GPL) and the Berkeley Software Distribution (BSD) License. Although the above studies relate to commercial open source, they do not analyze the impact of commercial open source software on the competitive strategies for proprietary software providers and social welfare.

Proprietary software producers face many challenges in growing their business in today's competitive industry environment, one of which comes from commercial open source software. Since the property of owners for community and commercial open source software is entirely different, this may lead to the strategic reactions of proprietary software producers diverse when compete with them. However, most of the studies on the competition between proprietary and open source software do not consider commercial open source software, and the analysis of commercial open source business models do not involve the competitive strategies of proprietary software producers. From the perspective of commercial software providers, they want to know about how the presence of commercial open source software affects their strategic choices for competing successfully. From the viewpoint of social planners, they need to know about the impact of commercial open source software on the consumer surplus and social welfare. For these reasons, this paper considers how commercial open source software can play influences on the pricing (market share and profit) of proprietary software producers, consumer surplus and social welfare.

The rest of the paper is organized as follows. Section 2 presents the basic model. Section 3 analyzes the case that commercial open source software appears in the market. Section 4 compares the optimal solutions. The final section concludes this paper.

\section{The basic setup}

There are two types of software in a market: community open source software and proprietary software. Community open source software is from the not-for-profit community and proprietary software is from the commercial software producer. The quality of a software product depends on the level of usability (includes the ease of installation, documentation, user interface and the level of technical support) and functional quality (includes feature set, security, reliability, etc) (Choudhary \& Zhou, 2007; Kumar et al., 2011). A main weakness of open source software is the low usability (CIO, 2002). It is generally less user friendly in 
contrast to proprietary software. Software users are indexed by their level of technical ability, and measured by parameter $\theta$. The total number of users is normalized to 1 and they are uniformly distributed over the unit interval $[0,1]$. As the approach of Choudhary and Zhou (2007), this study assumes the users with higher level of technical skills have lower $\theta$, while those with lower level of technical skills have higher $\theta$. A software user with lower technical skills has higher willingness to pay for software usability than a software user with higher technical skills.

The indirect utility functions for the generic software user at $\theta \in[0,1]$ when he/she uses community open source and proprietary software are respectively given by:

$$
\begin{aligned}
& u_{o}=\theta v_{o}+f_{o}-p_{o} \\
& u_{p}=\theta v_{p}+f_{p}-p_{p}
\end{aligned}
$$

In (1) and (2), $v_{o}$ and $v_{p}$ are the usability of community open source and proprietary software respectively, and satisfy $0<v_{o}<v_{p}$ (i.e., proprietary software is better than community open source software in the software usability); $f_{o}$ and $f_{p}$ are the functional quality of community open source and proprietary software respectively; $p_{o}$ and $p_{p}$ are the price of community open source and proprietary software respectively. Note that, since community open source software can be freely available from the open source community, its price is equal to zero (i.e., $p_{o}=$ $0)$.

According to (1) and (2), the marginal software user who is indifferent between depolying

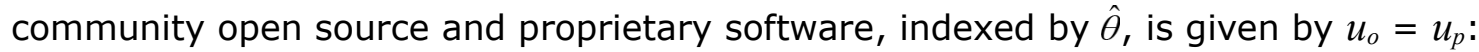

$$
\hat{\theta} v_{o}+f_{o}=\hat{\theta} v_{p}+f_{p}-p_{p}
$$

Solving equation (3) gives:

$$
\hat{\theta}=\frac{\Delta f+p_{p}}{v_{p o}}
$$

In (4), $\Delta f=f_{p}-f_{o}$ and $v_{p o}=v_{p}-v_{o}$.

Assume that the market is fully covered. Thus, the demand functions for open source community and proprietary software producer are respectively given by:

$$
\begin{gathered}
d_{o}=\frac{\Delta f+p_{p}}{v_{p o}} \\
d_{p}=1-\frac{\Delta f+p_{p}}{v_{p o}}
\end{gathered}
$$


The resulting profit function of proprietary software producer is:

$$
\pi_{p}=p_{p}\left(1-\frac{\Delta f+p_{p}}{v_{p o}}\right)=\frac{p_{p}\left(v_{p o}-\Delta f-p_{p}\right)}{v_{p o}}
$$

The social welfare is defined as the sum of the software producer's profit and consumer surplus. The consumer surplus function can be stated as:

$$
C S=\int_{0}^{\hat{\theta}}\left(\theta v_{o}+f_{o}\right) d \theta+\int_{\hat{\theta}}^{1}\left(\theta v_{p}+f_{p}-p_{p}\right) d \theta
$$

Thus, the welfare function corresponds to:

$$
S W=\pi_{p}+C S=\int_{0}^{\hat{\theta}}\left(\theta v_{o}+f_{o}\right) d \theta+\int_{\hat{\theta}}^{1}\left(\theta v_{p}+f_{p}\right) d \theta
$$

The proprietary software producer pursues profit maximization. The first-order condition of its profit function (7) yields the equilibrium price of proprietary software:

$$
p_{p}^{*}=\frac{v_{p o}-\Delta f}{2}
$$

The resulting equilibrium demands for open source community and proprietary software producer, and equilibrium profit for proprietary software producer are:

$$
\begin{gathered}
d_{o}^{*}=\frac{v_{p o}+\Delta f}{2 v_{p o}} \\
d_{p}^{*}=\frac{v_{p o}-\Delta f}{2 v_{p o}} \\
\pi_{p}^{*}=\frac{\left(v_{p o}-\Delta f\right)^{2}}{4 v_{p o}}
\end{gathered}
$$

Note that the demands or market shares of open source community and proprietary software producer must meet $0<d_{i}^{*}<1, i=o, p$. This requires $|\Delta f|<v_{p o}$.

Finally, the consumer surplus and social welfare are given by:

$$
\begin{gathered}
C S^{*}=\frac{1}{2}\left[\frac{\left(v_{p o}+\Delta f\right)\left(3 \Delta f-v_{p o}\right)}{4 v_{p o}}+\left(v_{p}+2 f_{p}\right)\right]-\frac{\left(v_{p o}-\Delta f\right)^{2}}{4 v_{p o}} \\
S W^{*}=\frac{1}{2}\left[\frac{\left(v_{p o}+\Delta f\right)\left(3 \Delta f-v_{p o}\right)}{4 v_{p o}}+\left(v_{p}+2 f_{p}\right)\right]
\end{gathered}
$$




\section{When proprietary software competes with both community and commercial open source software}

This section assumes that commercial open source software, which is provided by another software producer, appears in the market. Thus, proprietary software competes with both community and commercial open source software substitutes. In contrast to community open source software, its commercial version is owned by an entity with the purpose of reaping profits from the software. When commercial open source producers build software products based on publicly available source codes, they need to follow the corresponding open source licenses. The General Public License (GPL) is the most common open source license (Laurent, 2004). This License requires that software developers must open their development of features. However, they can keep their usability enhancements private under this license. For example, Red Hat Inc makes great contributions to Linux Kernel, Linux X Windows System and others, which must make publicly available under the GPL. Moreover, it provides users with additional services (includes extensive documentation, installation and maintenance, and technical support) that are available to users who buy its commercial product (Software Development Times, 2008; Kumar et al., 2011). This study only considers the case that commercial open source producer develops commercial software products based on community open source software under the GPL.

The indirect utility function for the generic user at $\theta \in[0,1]$ when he/she adopts commercial open source software is given by:

$$
u_{c}=\theta v_{c}+f_{c}-p_{c}
$$

In (16), $v_{c}$ is the usability of commercial open source software and meets $v_{o}<v_{c}<v_{p}$ (i.e., commercial open source software is better than community open source software and inferior to proprietary software in the software usability); $p_{c}$ is the price of commercial open source software; $f_{c}$ is the functional quality of commercial open source software. Note that, since open source community and commercial open source producer can wholly obtain each other's feature contributions under the GPL, their functional quality is assumed to be equal (i.e., $f_{c}=f_{o}$ ).

The marginal user who is indifferent between deploying community and commercial open source software, indexed by $\hat{\theta}$, is given by $u_{o}=u_{c}$ :

$$
\hat{\theta} v_{o}+f_{o}=\hat{\theta} v_{c}+f_{c}-p_{c}
$$

Thus,

$$
\hat{\theta}=\frac{p_{c}}{v_{c o}}
$$


Where $v_{c o}=v_{c}-v_{o}$.

The marginal user who is indifferent between adopting commercial open source and

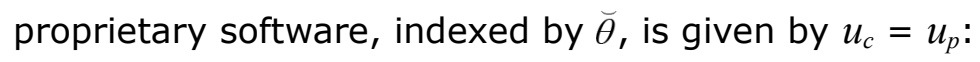

$$
\breve{\theta} v_{c}+f_{c}-p_{c}=\breve{\theta} v_{p}+f_{p}-p_{p}
$$

Solving the equation (19) yields:

$$
\widetilde{\theta}=\frac{\Delta f+p_{p}-p_{c}}{v_{p c}}
$$

Where $v_{p c}=v_{p}-v_{c}$.

According to (18) and (20), the demand functions for open source community, commercial open source and proprietary software producers are respectively given by:

$$
\begin{gathered}
d_{o}=\hat{\theta}-0=\frac{p_{c}}{v_{c o}} \\
d_{c}=\breve{\theta}-\widehat{\theta}=\frac{\Delta f+p_{p}-p_{c}}{v_{p c}}-\frac{p_{c}}{v_{c o}} \\
d_{p}=1-\breve{\theta}=1-\frac{\Delta f+p_{p}-p_{c}}{v_{p c}}
\end{gathered}
$$

The profit functions for commercial open source and proprietary software producers, and the consumer surplus function are respectively given by:

$$
\begin{gathered}
\pi_{c}=p_{c} d_{c}=p_{c}\left(\frac{\Delta f+p_{p}-p_{c}}{v_{p c}}-\frac{p_{c}}{v_{c o}}\right) \\
\pi_{p}=p_{p} d_{p}=p_{p}\left(1-\frac{\Delta f+p_{p}-p_{c}}{v_{p c}}\right) \\
C S=\int_{0}^{\hat{\theta}}\left(\theta v_{o}+f_{o}\right) d \theta+\int_{\hat{\theta}}^{\breve{\theta}}\left(\theta v_{c}+f_{c}-p_{c}\right) d \theta+\int_{\breve{\theta}}^{1}\left(\theta v_{p}+f_{p}-p_{p}\right) d \theta
\end{gathered}
$$

Adding up the profits of software producers and the consumer surplus gives the social welfare function:

$$
S W=\pi_{p}+\pi_{c}+C S=\int_{0}^{\hat{\theta}}\left(\theta v_{o}+f_{o}\right) d \theta+\int_{\hat{\theta}}^{\breve{\theta}}\left(\theta v_{c}+f_{c}\right) d \theta+\int_{\bar{\theta}}^{1}\left(\theta v_{p}+f_{p}\right) d \theta
$$

Both proprietary and commercial open source producers pursue profit maximization. The firstorder conditions of their profit functions give:

$$
\frac{\partial \pi_{c}}{\partial p_{c}}=\frac{\Delta f+p_{p}-2 p_{c}}{v_{p c}}-\frac{2 p_{c}}{v_{c o}}=0
$$




$$
\frac{\partial \pi_{p}}{\partial p_{p}}=1-\frac{\Delta f+2 p_{p}-p_{c}}{v_{p c}}=0
$$

Solving (28) and (29) yields the equilibrium prices for commercial open source and proprietary software:

$$
\begin{gathered}
p_{c}^{* \prime}=\frac{v_{c o}\left(v_{p c}+\Delta f\right)}{4 v_{p o}-v_{c o}} \\
p_{p}^{* \prime}=\frac{2 v_{p o} v_{p c}-\left(2 v_{p o}-v_{c o}\right) \Delta f}{4 v_{p o}-v_{c o}}
\end{gathered}
$$

The resulting demands, profits, consumer surplus and social welfare are given by:

$$
\begin{gathered}
d_{o}^{* \prime \prime}=\frac{v_{p c}+\Delta f}{4 v_{p o}-v_{c o}} \\
d_{c}^{* \prime \prime}=\frac{v_{p o}\left(v_{p c}+\Delta f\right)}{v_{p c}\left(4 v_{p o}-v_{c o}\right)} \\
d_{p}^{* \prime \prime}=\frac{2 v_{p o} v_{p c}-\left(2 v_{p o}-v_{c o}\right) \Delta f}{v_{p c}\left(4 v_{p o}-v_{c o}\right)} \\
\pi_{c}^{* \prime}=\frac{v_{c o} v_{p o}\left(v_{p c}+\Delta f\right)^{2}}{v_{p c}\left(4 v_{p o}-v_{c o}\right)^{2}} \\
C S^{* \prime \prime}=\frac{\left[2 v_{p o} v_{p c}-\left(2 v_{p o}-v_{c o}\right) \Delta f\right]^{2}}{v_{p c}\left(4 v_{p o}-v_{c o}\right)^{2}} \\
+\left(-v_{c o}\left(\frac{v_{p c}+\Delta f}{4 v_{p o}-v_{c o}}\right)^{2}+\frac{\left[\left(6 v_{p o}-v_{c o}\right) \Delta f-\left(2 v_{p o}-v_{c o}\right) v_{p c}\right]\left(2 v_{p o}-v_{c o}\right)\left(v_{p c}+\Delta f\right)}{v_{p c}\left(4 v_{p o}-v_{c o}\right)^{2}}\right. \\
\left.+\left(v_{p}+2 f_{p}\right)\right\}-\frac{v_{c o} v_{p o}\left(v_{p c}+\Delta f\right)^{2}+\left[2 v_{p o} v_{p c}-\left(2 v_{p o}-v_{c o}\right) \Delta f\right]^{2}}{v_{p c}\left(4 v_{p o}-v_{c o}\right)^{2}} \\
S W^{* \prime \prime}=\frac{1}{2}\left\{-v_{c o}\left(\frac{v_{p c}+\Delta f}{4 v_{p o}-v_{c o}}\right)^{2}+\frac{\left[\left(6 v_{p o}-v_{c o}\right) \Delta f-\left(2 v_{p o}-v_{c o}\right) v_{p c}\right]\left(2 v_{p o}-v_{c o}\right)\left(v_{p c}+\Delta f\right)}{v_{p c}\left(4 v_{p o}-v_{c o}\right)^{2}}\right. \\
\left.+\left(v_{p}+2 f_{p}\right)\right\}
\end{gathered}
$$

Note that the demands or market shares of open source community, and commercial open source and proprietary software producers must satisfy $0<d_{i}^{*}<1, i=o, c, p$. This requires $-v_{p c}<\Delta f<\frac{2 v_{p o} v_{p c}}{2 v_{p o}-v_{c o}}$ 


\section{Comparison}

According to the optimal results in section 2 and section 3, we can analyze how commercial open source software impacts the equilibrium price (demand and profit) of proprietary software producer, consumer surplus and social welfare.

Proposition 1. (i) $p_{p}^{*}>p_{p}^{*}$; (ii) when $\Delta f>\frac{v_{p c} v_{p o}}{3 v_{p o}-v_{c o}}, d_{p}^{*}>d_{p}^{*}$; when $\Delta f<\frac{v_{p c} v_{p o}}{3 v_{p o}-v_{c o}}, d_{p}^{*}<d_{p}^{*}$; $\pi_{p}^{*}>\pi_{p}^{* \prime}$

Proof. (i) since $|\Delta f|<v_{p o r} p_{p}^{* \prime}-p_{p}^{*}=-\frac{v_{c o}\left(3 v_{p o}-\Delta f\right)}{2\left(4 v_{p o}-v_{c o}\right)}<0$; (ii) since $d_{p}^{* \prime}-d_{p}^{*}=v_{c o} \frac{v_{p o} v_{p c}-\left(3 v_{p o}-v_{c o}\right) \Delta f}{v_{p c}\left(4 v_{p o}-v_{c o}\right)}, d_{p}^{*}>d_{p}^{* \prime}$ holds if $\Delta f>\frac{v_{p c} v_{p o}}{3 v_{p o}-v_{c o}}, \quad$ and $d_{p}^{*}<d_{p}^{*} \quad$ holds $\quad$ if $\Delta f<\frac{v_{p c} v_{p o}}{3 v_{p o}-v_{c o}} ; \quad$ (i i i ) since $\Delta f>-v_{p c}>-\frac{\sqrt{v_{p c}} v_{p o}\left[\left(4 v_{p o}-v_{c o}\right)-4 \sqrt{v_{p o} v_{p c}}\right]}{2 \sqrt{v_{p o}}\left(2 v_{p o}-v_{c o}\right)-\sqrt{v_{p c}}\left(4 v_{p o}-v_{c o}\right)}, \sqrt{\frac{\pi_{p}^{* *}}{\pi_{p}^{*}}}=\frac{2 \sqrt{v_{p o}}\left[2 v_{p o} v_{p c}-\left(2 v_{p o}-v_{c o}\right) \Delta f\right]}{\sqrt{v_{p c}}\left(4 v_{p o}-v_{c o}\right)\left(v_{p o}-\Delta f\right)}<1$ holds. Thus, $\pi_{p}^{*}>\pi_{p}^{* \prime}$.

In contrast to the case of proprietary software only competing with community open source software, the equilibrium price of proprietary software is lower when commercial open source software presents in the market. The intuition of this result is as follows. The commercial open source software's price is generally very low and its software usability is very high (whose usability is closer to proprietary software than community open source software). Such lowprice and high-usability software forces proprietary software producer to cut its price.

The impact of commercial open source software on the demand and profit for proprietary software producer depends on the relative difference in the level of functional quality between open source and proprietary software (i.e., $\Delta f$ ). If the difference is large enough, the equilibrium demand for proprietary software producer when commercial open source software presents is less than when it does not. If the difference is small enough, the opposite situation may appear. This result is evident in the operating system markets. In server operating system market, the market share of Windows significantly declines along with the development of commercial open source Linux. While in desktop operating system market, the market share of Windows does not obviously decrease despite more and more commercial open source products appear. According to the third part of Proposition 1, the appearance of commercial open source software can lead to the decrease of equilibrium profit of proprietary software producer. 
Corollary 1. When $f_{o}=f_{p}, d_{p}^{*}<d_{p}^{* \prime}$.

Proof. According to Proposition $1, d_{p}^{*}<d_{p}^{* \prime}$ holds if $\Delta f<\frac{v_{p c} v_{p o}}{3 v_{p o}-v_{c o}}$. Thus, $d_{p}^{*}<d_{p}^{*}$ if $\Delta f=0$. This corollary is thus proved.

When the functional quality of (both community and commercial) open source software is equal to proprietary software, the presence of commercial open source software will not lead to the decline in the share of proprietary software, instead, it rises. This is because the usability of commercial open source software is closer to proprietary software than community open source software. Therefore, the price competition is more intense when commercial open source software presents in the market than when it does not. Proprietary software producer will choose to lower its software price in order to attract more users. This finally leads to the increase of proprietary software producer's market share.

Proposition 2. When $f_{o}=f_{p}, C S^{* 1}>C S^{*}$ and $S W^{* \prime}>S W^{*}$.

Proof. When $f_{o}=f_{p}, C S^{* \prime}-C S^{*}=-\frac{\left(12 v_{p o}-v_{c o}\right) v_{p o} v_{p c}}{2\left(4 v_{p o}-v_{c o}\right)^{2}}+\frac{3 v_{p o}}{8}>0$

When $f_{o}=f_{p}, S W^{* \prime}-S W^{*}=\frac{1}{2}\left[-\frac{v_{c o} v_{p c}^{2}+\left(2 v_{p o}-v_{c o}\right)^{2} v_{p c}}{\left(4 v_{p o}-v_{c o}\right)^{2}}+\frac{v_{p o}}{4}\right]$.

Since $\frac{v_{c o} v_{p c}^{2}+\left(2 v_{p o}-v_{c o}\right)^{2} v_{p c}}{\left(4 v_{p o}-v_{c o}\right)^{2}}=\frac{\left(4 v_{p o}-3 v_{c c}\right) v_{p o} v_{p c}}{\left(4 v_{p o}-v_{c o}\right)^{2}}<\frac{\left(4 v_{p o}-v_{c o}\right) v_{p o} v_{p c}}{\left(4 v_{p o}-v_{c o}\right)^{2}}=\frac{v_{p o}\left(v_{p o}-v_{c o}\right)}{\left(4 v_{p o}-v_{c o}\right)}<\frac{v_{p o}}{4}, S W^{* \prime}-S W^{*}>0$.

When the functional quality of (both community and commercial) open source software is not equal to proprietary software (i.e., $f_{o} \neq f_{p}$ ), it is difficult to compare the values of $C S^{* 1}$ and $C S^{*}$ ( or $S W^{* \prime}$ and $S W^{*}$ ). However, the numerical analysis shows that, when $f_{o}>f_{p}$ or $f_{o}<f_{p}$, $C S^{* \prime}>C S^{*}$ and $S W^{* \prime}>S W^{*}$ are still found (see Figure 1 and Figure 2). That is, whether (both community and commercial) open source software is better than, equal to, or inferior to proprietary software in the functional quality, the appearance of commercial open source software can lead to the increase of the consumer surplus and social welfare. The reason is that, in contrast to proprietary software only competing with community open source software, when commercial open source software is also in the market, users have more types of software product for choices and the price of proprietary software declines. This results in the increase of the consumer surplus and social welfare. Thus, from the perspective of social policy-makers, it needs to encourage the development of commercial open source software. 


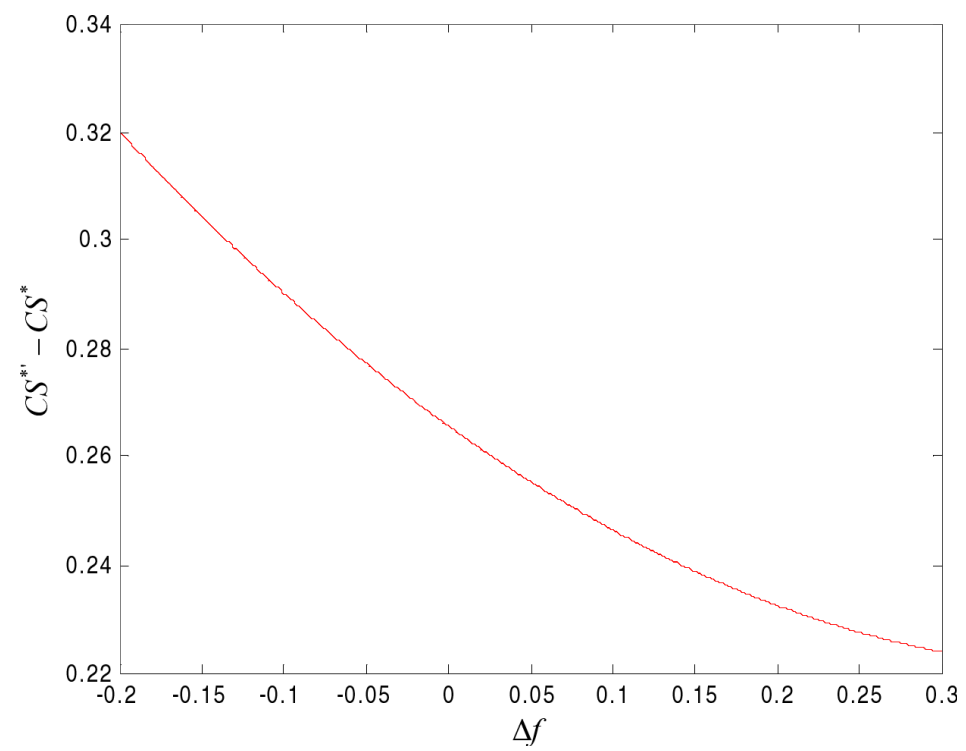

Figure 1. The value of $C S^{* \prime}-C S^{*}$ varies with $\Delta f$ (if $v_{o}=1, v_{c}=1.8$ and $v_{p}=2$ ).

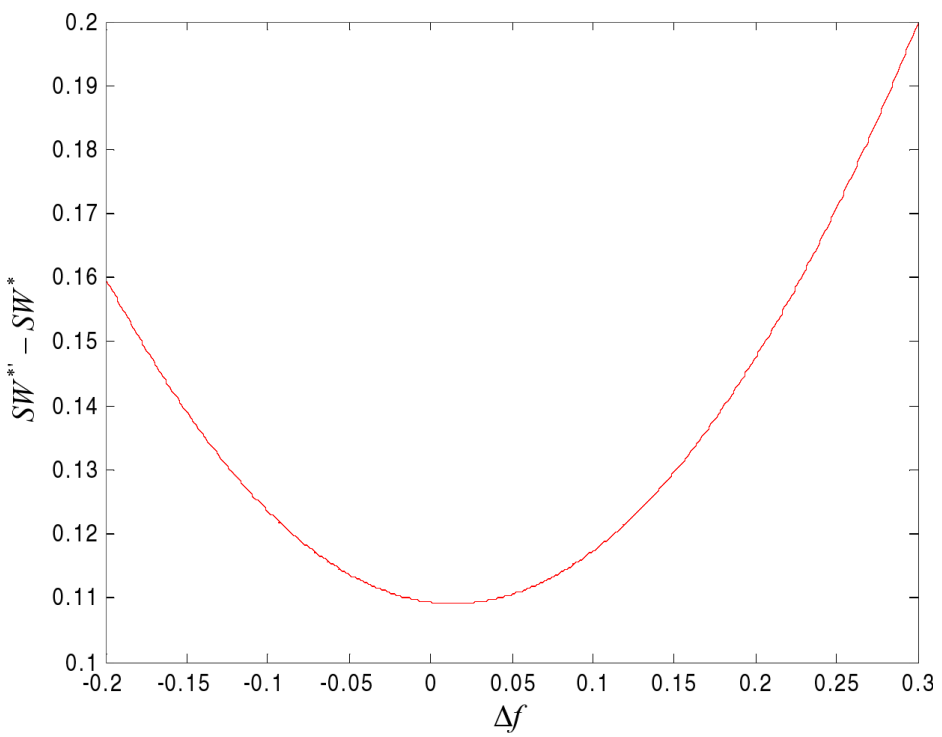

Figure 2. The value of $S W^{* \prime}-S W^{*}$ varies with $\Delta f$ (if $v_{o}=1, v_{c}=1.8$ and $v_{p}=2$ )

It is worth noting that the impact of community and commercial open source software on proprietary software producers and social welfare may be different. Lanzi (2009) shows that the emergence of community open source will increase the price of proprietary software when its users form a large but poorly skilled network. Xing (2010b) finds that, if the learning (maintenance or development) costs for community open source software are sufficiently high, the profit of proprietary software producer when it monopolizes the market is less than when it competes with community open source software. Choudhary and Zhou (2007) think that the competition from community open source software may reduce the social welfare when its quality is high enough. Thus, we need to distinguish between community and commercial open source software when study the competition between open source and proprietary software. 


\section{Conclusions}

By building two vertical-differentiation models, this study investigates the impact of competition from commercial open source software on proprietary software producer. It assumes that commercial open source software is better than community open source software and inferior to proprietary software in the usability and mainly finds that: (i) in contrast to the case of proprietary software only competing with community open source software, the equilibrium price and profit of proprietary software producer is lower when the market also exists commercial open source software; (ii) if the difference in the functional quality between open source and proprietary software is large (resp. small) enough, the equilibrium demand of proprietary software producer is lower (resp. higher) when commercial open source software presents than when it does not; (iii) the presence of commercial open source software can lead to the increase of the consumer surplus and social welfare.

\section{Acknowledgements}

We acknowledge the financial support from Natural Science Foundation of Shandong Province (No. ZR2013GL005), Social Science Planning Research Project of Shandong Province (No. 12CJRJ17), Shandong province soft science research plan (No. 2014RKB01165) and Shandong Higher School of Humanities and Social Science Research Projects (No. J13WF11).

\section{References}

Chen, H.K., Liu, Y., \& Tang, Q. (2011). The impact of network externalities on the competition between open source and proprietary software. Journal of Management Information Systems, 27(4), 201-230. http://dx.doi.org/10.2753/MIS0742-1222270407

Choudhary, V., \& Zhou, Z.Z. (2007). Impact of competition from open source software on proprietary software. Working Paper Series No. 08070, China Economics and Management Academy. http://dx.doi.org/10.1596/1813-9450-4102

Coar, K. (2006). The open source definition (annotated). Available at: http://www.opensource.org/docs/definition.php

CIO. 2002. Open source gains momentum.

Deshpande, A., \& Riehle, D. (2008). The total growth of open source. The International Federation for Information Processing, 275, 197-209.

Dixon, J. (2009). The beekeeper model of commercial open source software. Available at: http://wiki.pentaho.com/display/BEEKEEPER/The+Beekeeper 
Economides, N., \& Katsamakas, E. (2006). Two-sided competition of proprietary vs. open source technology platforms and the implications for the software industry. Management Science, 52(7), 1057-1071. http://dx.doi.org/10.1287/mnsc.1060.0549

Gramstad, A.R. (2014). Piracy in commercial vs. open-source software competition. Working Paper. Department of Economics, University of Oslo.

Jaisingh, J., See-To, E., \& Tam, K. (2008). The impact of open source software on the strategic choices of firms developing proprietary software. Journal of Management Information Systems, 25(3), 241-276. http://dx.doi.org/10.2753/MIS0742-1222250307

Kumar, V., Gordon, B., \& Srinivasan, K. (2011). Competitive strategy for open source software. Marketing Science, 30(6), 1066-1078. http://dx.doi.org/10.1287/mksc.1110.0669

Lanzi, D. (2009). Competition and open source with perfect software compatibility. Information Economics and Policy, 21(3), 192-200. http://dx.doi.org/10.1016/j.infoecopol.2008.11.004

Laurent, L.S. (2004). Understand op en source and fre e software licencing. Cambridge, Massachsusetts: O'Reilly. Available at: https://people.debian.org/ dktrkranz/legal/Understanding \%200pen\%20Source\%20and\%20Free\%20Software\%20Licensing.pdf

Lee, D., \& Mendelson, H. (2008). Divide and conquer: competing with free technology under network effects. Production and Operations Management, 17(1), 12-28. http://dx.doi.org/10.3401/poms.1070.0005

Lin, L.H. (2008). Impact of user skills and network effects on the competition between open source and proprietary software. Electronic Commerce Research and Applications, 7(1), 68-81. http://dx.doi.org/10.1016/j.elerap.2007.01.003

O'Reilly, T. (1999). Lessons from open-source software development. Communications of the ACM, 42(2), 33-37.

Popp, K.M. (2012). Leveraging open source licenses and open source communities in hybrid commercial open source business models. Proceedings of the Fourth International Workshop on Software Ecosystems (IWSECO 2012), Boston, MA, USA.

Raghunathan, S., Prasad, A., Mishra, B.K., \& Chang, H. (2005). Open source versus closed source: software quality in monopoly and competitive markets. IEEE Transactions on Systems Man and Cybernetics Part A Systems and Humans, 35(6), 903-918. http://dx.doi.org/10.1109/TSMCA.2005.853493

Riehle, D. (2012). The single-vendor commercial open source business model. Information Systems and e-Business Management, 10(1), 5-17. http://dx.doi.org/10.1007/s10257-010-0149-x 
Software Development Times (2008). Red hat tops list of corporate linux code contributors. Available at: http://truthhappens.redhat.com/?s=contributor

Sen, R. (2007). A strategic analysis of competition between open source and proprietary software. Journal of Management Information Systems, 24(1), 238-258. http://dx.doi.org/10.2753/MIS0742-1222240107

Widenius, W., \& Nyman, N. (2014). The business of open source software: a primer. Technology Innovation Management Review, 1, 8-11.

Xing, M.Q. (2010a). Proprietary software's R\&D decisions when open source software appears in a software industry. The International Conference on E-Product, E-Service and E-Entertainment, 1518-1521.

Xing, M.Q. (2010b). Does open source software decrease profit of proprietary software producer and increase social welfare. The 2nd International Conference on Information Science and Engineering (ICISE 2010), 203-206.

Xing, M.Q. (2013). Comparative study on innovation incentives for commercial open source software under different licenses. Research Journal of Applied Sciences, Engineering and Technology, 5(5), 1633-1638.

Zeroukhi, M., \& Pénard, T. (2014). Open source software subsidies and network compatibility in a mixed duopoly. Economics Bulletin, 34(2), 1174-1184.

Zhu, K., \& Zhou, Z. (2012). Lock-in strategy in software competition: Open-source software vs. proprietary software. Information Systems Research, 23(2), 536-545.

http://dx.doi.org/10.1287/isre.1110.0358

Journal of Industrial Engineering and Management, 2014 (www. jiem. org)

Article's contents are provided on a Attribution-Non Commercial 3. 0 Creative commons license. Readers are allowed to copy, distribute and communicate article's contents, provided the author's and Journal of Industrial Engineering and Management's names are included. It must not be used for commercial purposes. To see the complete license contents, please visit http://creativecommons. org/licenses/by-nc/3. 0\%. 